\title{
Características motivacionais de atletas brasileiros
}

\author{
Motivational characteristics of Brazilian athletes
}

\author{
D.R. Coimbra, S.S. Gomes, H.Z. Oliveira, R. A. Rezende, D. Castro, R. Miranda, M.G. Bara \\ Filho
}

ARTIGO ORIGINAL | ORIGINAL ARTICLE

\begin{abstract}
RESUMO
O objetivo do presente estudo foi verificar diferenças e semelhanças nas características motivacionais de atletas brasileiros com base na Teoria da Autodeterminação (Deci \& Ryan, 1985), comparando desportistas de diferentes idades, modalidades coletivas e individuais, de ambos os sexos, em distintos níveis de rendimento. A amostra foi constituída por 344 atletas, com uma idade média de $24.3 \pm 6.0$ anos. A maioria era do sexo masculino (64.5\%), de desportos coletivos (59.9\%) e que competiam em nível nacional e internacional (64.1\%). Aplicou-se o Sport Motivation Scale (SMS), validado em português (SMS-Br) para medir os níveis de motivação. Foram encontradas diferenças estatisticamente significativas nas dimensões Motivação Extrínseca de Introjeção, Motivação Extrínseca de Regulação Externa e Amotivação, na comparação feita por género. Também foram encontradas diferenças estatísticas nas dimensões Motivação Intrínseca para Conseguir e Motivação Extrínseca de Introjeção, ao comparar o tipo de desporto. Os resultados sugerem que atletas brasileiros possuem características motivacionais únicas ao serem comparados com atletas de diferentes países.

Palavras-chave: motivação, autodeterminação, género, desporto competitivo
\end{abstract}

ABSTRACT

The aim of this study was to determine differences and similarities in the motivational characteristics of Brazilian athletes based on Self-Determination Theory (Deci \& Ryan, 1985). It was analyzed differences between gender (male and female) and type of sport (individual and collective). The sample consisted of 344 athletes, mean age $24.3 \pm 6.0$. The most subjects were males $(64.5 \%)$, engaged in team sports $(59.9 \%)$ and competing at national and international level $(64.1 \%)$. It was applied the Sport Motivation Scale (SMS), validated in Portuguese (Br-SMS) to measure levels of motivation. There were significant differences in the dimensions Extrinsic Motivation to Introjection, Extrinsic Motivation to External Regulation and Amotivation, according to gender. It was also found significant differences in the dimensions Intrinsic Motivation to Toward Accomplish and Extrinsic Motivation the Introjection according to the sport. The results suggest that Brazilian athletes have unique motivational characteristics when compared with athletes from other countries.

Keywords: motivation, self-determination, gender, competitive sport

Submetido: 16.07.2012 | Aceite: 14.03.2013

Danilo Reis Coimbra, Simone Salvador Gomes, Helder Zimmermann de Oliveira, Rafael Andrade Rezende, Débora Castro, Renato Miranda, Mauricio Gattás Bara Filho. Faculdade de Educação Física e Desportos, Universidade Federal de Juiz de Fora, Minas Gerais, Brasil.

Endereço para correspondência: Helder Zimmermann de Oliveira, Faculdade de Educação Física e Desportos, Universidade Federal de Juiz de Fora, Rua José Lourenço Kelmer, S/N - Campus Universitário, Bairro São Pedro, CEP: 36036-900 Juiz de Fora - MG, Brasil.

E-mail: helderzimmermann@yahoo.com.br 
Os motivos que levam os indivíduos a praticar e a se manterem no desporto têm recebido grande atenção dos pesquisadores em Psicologia do Desporto em todo o mundo (Gomes, Coimbra, Garcia, Miranda, \& Bara Filho, 2007). Ao mesmo tempo, atletas e treinadores consideram importante o desenvolvimento de novos métodos para aumentar a motivação (Coimbra et al., 2008). Ou seja, tanto psicólogos do desporto, quanto treinadores e atletas concordam que a motivação é um fator de extrema importância no desporto competitivo.

Vários são os aspetos primordiais para o atleta atuar no desporto competitivo. Destacando o aspeto psicológico, o atleta necessita de um alto grau de motivação para suportar as cargas dos treinos, o estresse gerado durante as competições além da pressão de patrocinadores, familiares e treinadores. Deste modo, treinamentos e competições compatíveis com a capacidade do atleta provocarão um nível ótimo de motivação (Miranda \& Bara Filho, 2008).

A Teoria da Autodeterminação, postulada por Deci e Ryan (1985), estabelece que existam três necessidades psicológicas básicas e primordiais: competência, relação social e autonomia. A necessidade de competência é a experiência de controlar o resultado, ou seja, se sentir capaz de algo. A de relação social é o esforço para constituir relações afetivas com o próximo, preocupar-se com o outro e sentir reciprocidade. E a de autonomia é o desejo de se sentir na origem de suas ações (Fernandes \& Vasconcelos-Raposo, 2005; Murcia, Gimeno \& Coll, 2007; Murcia \& Coll, 2006; Perreault \& Vallerand, 2007; Ryan \& Deci, 2000). Esta teoria estabelece que a motivação do indivíduo seja vista de uma forma contínua, definida por níveis de autodeterminação, da motivação mais autodeterminada para a menos autodeterminada, resultando em três diferentes tipos de motivação: intrínseca, extrínseca e amotivação. (Chantal, Guay, Dobreva-Martinova, \& Vallerand, 1996; Cresswell \& Eklund, 2005; Perreault \& Vallerand, 2007; Sarrazin, Vallerand,
Guillet, Pelletier, \& Cury, 2002).

A motivação Intrínseca (MI) se refere à atividade que é praticada simplesmente pelo prazer e pela satisfação gerada pela própria atividade, ou seja, o indivíduo participa voluntariamente, sem esperar recompensas externas à atividade. Por exemplo, desportistas que sentem satisfação em aprender novas técnicas do seu desporto. Diversos autores afirmam que a motivação intrínseca pode ser dividida em três diferentes dimensões: MI para saber, MI para realizar e MI para estimulação da experiência (Fernandes \& Vasconcelos-Raposo, 2005; Murcia \& Coll, 2006; Murcia, Gimeno, et al., 2007; Nuñes, Martín-Balbo, Navarro, \& González, 2006; Pelletier et al., 1995; Perreault \& Vallerand, 2007; Ryan \& Deci, 2000; Vallerand et al., 1992).

Atletas são intrinsecamente motivados para saber quando têm prazer em aprender algo novo, como uma técnica nova, se satisfaz com a experiência em que ele aprende, explora e tenta realizar (Pelletier et al., 1995). A motivação intrínseca para realizar é quando a atividade experimentada gera prazer e satisfação na realização de algo. Tentar dominar uma técnica difícil no desporto, por exemplo (Pelletier et al., 1995). A motivação intrínseca para estimulação da experiência acontece quando se inicia uma atividade para experimentar as sensações derivadas da experiência, tais como prazer, divertimento, alegria (Pelletier et al., 1995).

A motivação extrínseca (ME) faz referência aos comportamentos já comprometidos como um meio para um fim, e não para a própria causa, por exemplo, atletas que participam de competições apenas para ganhar reconhecimento dos técnicos e familiares. Deci e Ryan (1985) propuseram que a ME pode ser ordenada de acordo com o contínuo da autodeterminação da forma mais autodeterminada para a menos autodeterminada, respetivamente: regulação integrada, identificada, introjetada e regulação externa.

A regulação integrada acontece quando várias identificações são assimiladas e organi- 
zadas hierarquicamente, ou seja, os motivos são classificados e alocados com outros valores e necessidades.

A regulação identificada se refere ao comportamento autónomo, porém a decisão de realizar a tarefa é dada pelos benefícios externos e não pelo simples prazer e satisfação inerente a própria atividade.

A introjeção implica evitar os sentimentos ruins, tais como culpa e ansiedade e agregar expectativas de autoaprovação e orgulho, através do estabelecimento de regras e deveres para a ação.

A busca por incentivos externos à prática é o que caracteriza a regulação externa. O indivíduo se empenha na tarefa apenas para alcançar uma recompensa ou evitar uma punição.

Por último, a forma menos autodeterminada de motivação é a amotivação, em que o indivíduo não percebe mais importância nas suas ações, há um sentimento de incompetência e perda de controle (Fernandes \& Vasconcelos-Raposo, 2005; Murcia \& Coll, 2006; Murcia, Gimeno, et al., 2007; Nuñes, MartínBalbo, Navarro, \& González, 2006; Pelletier et al., 1995; Perreault \& Vallerand, 2007; Ryan \& Deci, 2000; Vallerand et al., 1992).

A fim de avaliar todas as formas de motivação de uma maneira quantitativa, Pelletier et. al. (1995) criaram o SMS, um questionário autoaplicável, possui uma escala tipo Likert de 1 até 7 pontos para cada uma das 28 questões, sendo 4 para cada dimensão da motivação. A partir da primeira validação deste questionário, o SMS foi amplamente utilizado em diversos estudos no contexto desportivo competitivo (Chantal et al., 1996); educacional (Fernandes \& Vasconcelos-Raposo, 2005) e da atividade física (Alexandris, Tsorbatzoudis, \& Grouios, 2002), validado para outras línguas (Chantal et al.,1996; Nuñes et al., 2006), aplicado conjuntamente com outros questionários (Gillet, 2008; Luján \& Deval, 2006; Murcia, Blanco, Galindo, Villodre, \& Coll, 2007; Perreault \& Vallerand, 2007) e utilizados para predizer e correlacionar com outras variáveis psicológicas. (Cresswell \& Eklund 2005; Lemyre, Roberts,
\& Stray-Gunderrsen, 2007; Sarrazin et al., 2002).

A motivação é um elemento chave para se conseguir a permanência e o prazer no desporto, além de ser determinante na conduta do atleta, pois desperta, dá energia e regula seu comportamento, dependendo de fatores pessoais e ambientais. Logo, o motivo para a prática desportiva varia de atleta para atleta. Entretanto, apesar dos estudos sobre motivação com atletas brasileiros, poucos analisaram suas diversas formas de motivação.

Diante das considerações acima citadas, o objetivo do presente estudo foi verificar diferenças e semelhanças nas características motivacionais de atletas brasileiros com base na Teoria da Autodeterminação, comparando desportistas de diferentes idades, modalidades coletivas e individuais, de ambos os sexos, em distintos níveis de rendimento.

\section{MÉTODO}

\section{Participantes}

Composta em sua maioria por atletas do género masculino $(64.5 \%)$, praticantes de modalidades coletivas $(59.9 \%)$, com experiência em competições de nível nacional/internacional $(64.1 \%)$, com idades entre os 14 e os 47 anos (63\% acima de 21 anos) e tempo de experiência na modalidade variando de 1 a 33 anos (60\% acima de 6 anos). Na Tabela 1 são apresentadas as características gerais da amostra do estudo.

\section{Instrumentos}

Aplicou-se a versão validada para a língua portuguesa por Bara Filho et al. (2011) da SMS de Pelletier et al. (1995), a qual passou a ser denominada por Escala de Motivação Esportiva (SMS-Br). Esta escala é enunciada pela seguinte pergunta "Porque você pratica esporte?" e avalia os diferentes tipos de motivação estabelecidos pela Teoria da Autodeterminação: MI para saber (ex. "Pelo prazer de descobrir novas técnicas de treinamento"), MI para realizar (ex. "Pela satisfação que sinto quando estou melhorando as minhas 
Tabela 1

Características gerais da amostra utilizada no estudo $(n=344)$

\begin{tabular}{lc}
\hline Variáveis & $\mathrm{n}(\%)$ \\
\hline Género & $222(64.5)$ \\
$\quad$ Masculino & $122(35.5)$ \\
$\quad$ Feminino & \\
Tipo de Desporto & $138(40.1)$ \\
$\quad$ Individual & $206(59.9)$ \\
$\quad$ Coletivo & \\
Nível Competitivo & $124(35.9)$ \\
$\quad$ Regional & $221(64.1)$ \\
$\quad$ Nacional/Internacional & $\mathrm{M} \pm \mathrm{DP}$ \\
\hline $\begin{array}{l}\text { Idade (anos) } \\
\text { Tempo de Experiência } \\
\text { (anos) }\end{array}$ & $24.3 \pm 6.0$ \\
\hline
\end{tabular}

habilidades"), MI para estimulação de experiência (ex. "Porque gosto da sensação de estar totalmente envolvido na atividade"), ME identificada (ex. "Porque o esporte é uma das melhores maneiras para manter boas relações com meus amigos/as"), ME introjeção (ex. "Porque eu devo praticar esportes regularmente"), ME regulação externa (ex. "Pelo prestígio de ser um atleta") e Amotivação ("Já não está tão claro para mim; na verdade, não acho que meu lugar é no esporte."). É composta de quatro itens para cada dimensão da motivação, sendo um total de 28 itens, que foram respondidas em uma escala tipo Likert (1: Não corresponde em nada; a 7: Corresponde exatamente). A versão brasileira apresentou valores de alpha de Cronbach variando de .68 para Amotivação até .81 para MI para saber.

\section{Procedimentos}

Primeiramente, o projeto de pesquisa foi aprovado pelo Comitê de Ética em Pesquisa da Universidade Federal de Juiz de Fora. Após, os treinadores e clubes foram contatados para aplicar o questionário nos atletas, informando sobre a voluntariedade na participação da pesquisa assim como assegurando o total sigilo e a garantia do anonimato do mesmo. Dois pes- quisadores administraram o questionário de acordo com instruções padronizadas. Não houve limite de tempo para o atleta preencher o SMS-Br.

A presente pesquisa atendeu às determinações da Declaração de Helsínquia e a resolução 196/96 do Conselho Nacional de Saúde.

\section{Análise Estatística}

Para a análise dos dados utilizou-se inicialmente a estatística descritiva (média e desvio padrão) para cada variável.

Posteriormente a significância dos fatores "Género" (masculino/feminino) e "Tipo de Desporto" (individual/coletivo) sobre a motivação para o desporto foi avaliada por uma MANCOVA $2 \times 2$, depois de validados os pressupostos de normalidade univariada de cada uma das variáveis dependentes (teste de Komolgorov-Smirnov), bem como a homogeneidade das matrizes de variância-covariância (teste $M$ de Box). Considerou-se apenas o "tempo de experiência desportiva" como covariável, uma vez que esta apresentou correlação significativa com a variável "idade" $(r=.63, p$ $<.01$ ). Quando a MANCOVA detetou efeitos estatisticamente significativos, procedeu-se a comparação múltipla das médias, ajustada pelo teste de Bonferroni. A análise do valor da estatística $F$ foi feita a partir do Traço de Pillai, considerando-se um nível de significância $\alpha=$ .05 .

\section{RESULTADOS}

A análise multivariada de covariância (MANCOVA) mostrou que existe um efeito significativo dos fatores (género e tipo de desporto) sobre os motivos de participação no desporto (motivação) improvável de ter ocorrido apenas por erro amostral. Quando controlada pela influência da variável tempo de experiência na modalidade, a análise resultou em efeitos principais significativos para género $\left(F_{(7,333)}=4.078, p=.001, \eta^{2}=.079 ;\right.$ poder $=$ $.987)$ e tipo de desporto $\left(F_{(7,333)}=2.252, p=\right.$ $.03 ; \eta^{2}=.045$; poder $=.83$ ), não havendo efeito significativo para a interação género $\times$ 
tipo de desporto $\left(F_{(7,333)}=1.617, p=.13 ; \eta^{2}=\right.$ .033 ; poder $=.67$ ).

Na tabela 2 são apresentadas as médias e desvios-padrão ajustados das variáveis de motivação para os fatores género e tipo de desporto. Quanto ao efeito do fator género, a análise univariada de comparação múltipla das médias mostrou diferenças significativas para as variáveis ME Introjetada $\left(F_{(1,339)}=4.599, p\right.$ $=.03, \eta^{2}=.013$; poder $\left.=.57\right)$, ME Externa $(F$ $(1,339)=19.166, p=.001 ; \eta^{2}=.054 ;$ poder $=$ $.99)$ e Amotivação $\left(F_{(1,339)}=4.949, p=.03, \eta^{2}\right.$ $=.014$, poder $=.60)$. O efeito observado é de pequena-média dimensão, com maior potência do teste observada para a variável ME Externa.

$\mathrm{Na}$ figura 1 observa-se que os atletas do género masculino apresentaram maiores escores de ME Introjetada $(5.05 \pm 1.45$ vs. $4.08 \pm$ 1.42), maior ME Externa (3.95 \pm 1.49 vs. 2.96 $\pm 1.28)$ e também maior Amotivação (2.23 \pm 1.31 vs. $1.89 \pm 1.14$ ) quando comparados às atletas do género feminino. Para as demais variáveis de motivação não foram observadas diferenças significativas na comparação entre géneros.

Quanto ao efeito do fator tipo de desporto, a análise univariada de comparação múltipla das médias mostrou diferenças significativas para as variáveis MI Conseguir $\left(F_{(1,339)}=\right.$ 4.897, $p=.03 ; \eta^{2}=.014$; poder $\left.=.60\right)$ e $\mathrm{ME}$ Introjetada $\left(F_{(1,339)}=9.852, p=.002, \eta^{2}=\right.$
.028 ; poder $=.88)$. Relativamente ao fator tipo de desporto, o efeito observado é de pequena dimensão, com maior potência do teste observada para a variável ME Introjetada.

$\mathrm{Na}$ figura 2, observa-se que os atletas de desportos individuais apresentaram maiores escores de MI Conseguir $(5.55 \pm 1.14$ vs. 5.04 $\pm 1.51)$ e maior ME Introjetada $(5.19 \pm 1.33$ vs. $4.38 \pm 1.53$ ) quando comparados aos de modalidades coletivas. Para as demais variáveis de motivação não foram observadas diferenças significativas entre os participantes de modalidades individuais e coletivas.

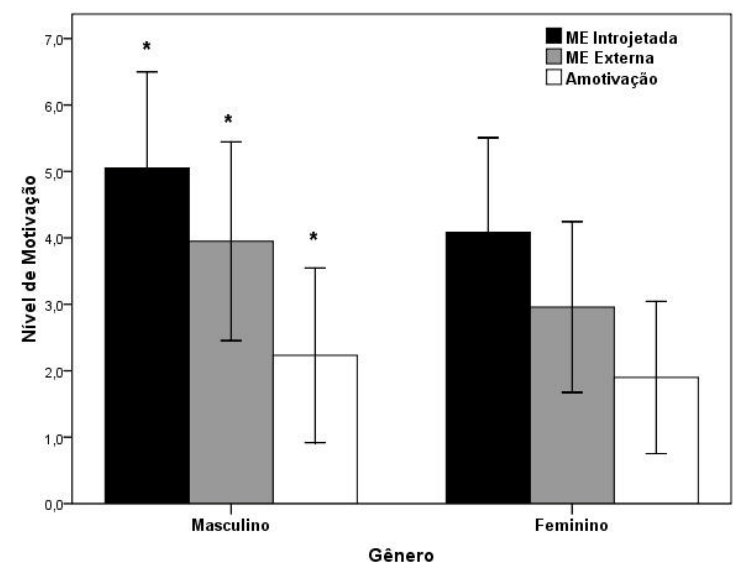

Figura 1. Média \pm desvio-padrão para os níveis de motivação externa (ME) e amotivação em atletas do género masculino e feminino $(n=344)$, considerando o tempo de experiência como covariável $=9.71$;

*diferenças significativas entre os géneros, $p<.05$

Tabela 2

Média e desvio-padrão das variáveis de motivação de atletas masculinos e femininos praticantes de modalidades desportivas individuais e coletivas $(n=344)$

\begin{tabular}{lcccc}
\hline \multirow{2}{*}{ Motivação } & \multicolumn{2}{c}{ Atletas Masculino } & \multicolumn{2}{c}{ Atletas Feminino } \\
& Desporto Individual & Desporto Coletivo & Desporto Individual & Desporto Coletivo \\
\hline MI Saber & $5.55 \pm 1.19$ & $5.04 \pm 1.62$ & $5.06 \pm 1.46$ & $5.05 \pm 1.40$ \\
MI Conseguir & $5.55 \pm 1.17$ & $5.07 \pm 1.55$ & $5.54 \pm 0.97$ & $5.01 \pm 1.48$ \\
MI Experiências & $5.89 \pm 0.98$ & $5.86 \pm 1.21$ & $6.07 \pm 0.82$ & $5.57 \pm 1.20$ \\
ME Identificada & $5.26 \pm 1.32$ & $5.13 \pm 1.51$ & $4.93 \pm 0.94$ & $4.46 \pm 1.51$ \\
ME Introjetada & $5.21 \pm 1.33$ & $4.85 \pm 1.55$ & $5.03 \pm 1.34$ & $3.93 \pm 1.38$ \\
ME Externa & $3.95 \pm 1.39$ & $3.94 \pm 1.62$ & $2.96 \pm 1.19$ & $2.96 \pm 1.30$ \\
Amotivação & $2.26 \pm 1.38$ & $2.19 \pm 1.23$ & $1.80 \pm 1.06$ & $1.91 \pm 1.16$ \\
\hline
\end{tabular}




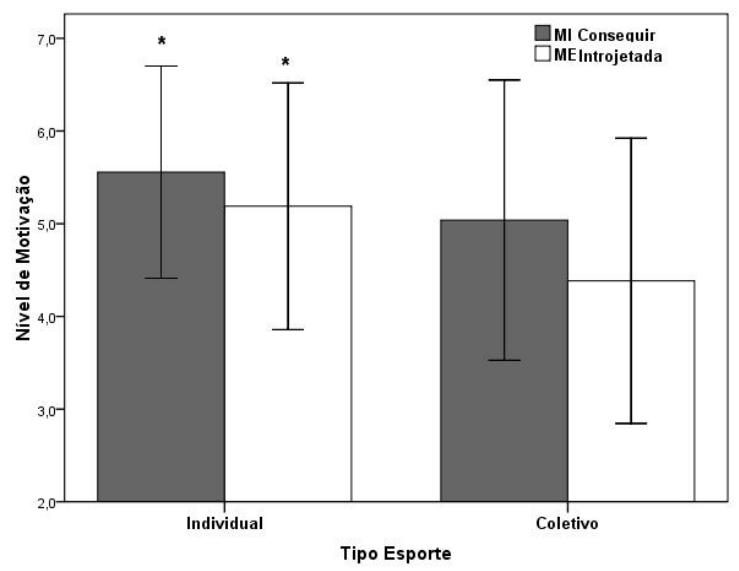

Figura 2. Média \pm desvio-padrão para os níveis de motivação interna (MI) e externa (ME) em atletas de modalidades individuais e coletivas $(n=344)$, considerando o tempo de experiência como covariável $=9.71 ;{ }^{*}$ diferenças significativas entre os tipos de desporto, $p<.05$

\section{DISCUSSÃO}

A proposta do trabalho foi avaliar as características motivacionais dos atletas brasileiros com base na Teoria da Autodeterminação através do questionário SMS-Br.

Tanto atletas do género feminino quanto do género masculino demonstraram níveis de $\mathrm{MI}$ e ME elevados e um nível baixo de amotivação. Porém, a média dos homens foi significativamente maior do que das mulheres para todas as variáveis. O único estudo que utilizou o mesmo instrumento, SMS validado para português também encontrou valores maiores em homens, para todas as dimensões. O estudo foi realizado em corredores de rua (Sena Júnior, 2012).

Em outro estudo realizado no Brasil com 417 estudantes entre 14 e 18 anos de uma escola pública de Florianópolis/SC, os resultados demonstraram que os rapazes foram mais autodeterminados para a prática de exercícios físicos do que as meninas (Silva, Matias, Viana, \& Andrade, 2012).

$\mathrm{O}$ artigo no qual se encontrou as propriedades psicométricas da versão espanhola do SMS em desportistas paraguaios revelou que todos os valores encontrados para as dimensões da autodeterminação encontraram valores maiores para homens exceto na ME de Introjeção que encontrou valores médios muito próximos (5.01 para homens e 5.04 para mulheres) (Martín-Balbo et al., 2007). Na mesma direção, com atletas universitários Amorose e Horn (2000) encontraram maiores valores de MI para homens.

Porém, em outro estudo com universitários e escolares, Amorose e Anderson-Butcher (2007) encontrou que as mulheres são mais autodeterminadas que os homens. Murcia, Blanco, et al. (2007) com jovens atletas e Gillet (2008), pesquisando atletas universitários de desportos competitivos e recreacionais, também encontraram valores na MI significativamente maiores nas atletas, indicando que as mulheres são mais autodeterminadas do que os homens.

Pelletier et al. (1995) encontrou níveis de MI maiores nas atletas do sexo feminino do que nos atletas do sexo masculino, porém em relação à ME os resultados encontrados mostraram que a ME de Regulação Externa é significativamente superior em atletas do sexo masculino.

Os resultados do presente estudo discordam também da pesquisa de Chantal et al. (1996) com atletas Búlgaros. Estes autores encontraram maior nível de MI nas atletas do género feminino, e ainda afirmaram que as mulheres praticam desporto mais para o prazer do que para obter outras recompensas externas.

Outros estudos acerca da relação género e motivação, foram realizados em culturas diferentes e demonstram que não existe um consenso acerca da relação género e dimensões da motivação segundo a teoria estudada (Fortier, Vallerand, Brière, \& Provencher, 1995; Halbrook, Blom, Hurley, Bell, \& Holden, 2012; Kingston, Horrocks \& Hanton, 2006; Krinanthi, Konstantinos, \& Andreas, 2010; Pero et al., 2009).

Os mais diversos resultados encontrados nos estudos demonstram que não existe uma relação direta entre género e as dimensões propostas pela autodeterminação. Em relação a atletas brasileiros, o maior nível em todas as 
dimensões para o sexo masculino pode ter ocorrido pelo fato de que no Brasil o desporto competitivo tem maior visibilidade para homens. A prática desportiva é menos estimulada para as mulheres, o que contribuiu para um menor número de atletas do sexo feminino participando da pesquisa e um nível menor de MI e ME. Para se ter uma ideia, a primeira medalha olímpica do Brasil no feminino foi ganha somente em Atlanta 1996. E, de acordo com Fernandes e Vasconcelos-Raposo (2005), devido à presença de estereótipos sexuais acerca de algumas modalidades existentes consideradas masculinas, as mulheres evidenciam menores níveis de MI, influenciando as perceções e pensamentos, limitando o empenho e a persistência nessas atividades.

Com relação ao nível competitivo, Chantal et al. (1996) compararam grupos de baixo e alto desempenho, usando como critério a performance dos atletas nas competições nos últimos dois anos. Na presente pesquisa, diferenciamos o nível competitivo dos atletas em relação ao nível das competições em que participavam (regionais, nacionais ou internacionais). Chantal et al. (1996) encontraram níveis de MI maiores em atletas de baixo desempenho, e níveis de ME maiores em atletas de alto desempenho, porém não foram identificadas diferenças significativas. O que difere do presente estudo, que encontrou maior grau de motivação tanto intrínseca quanto extrínseca em atletas de nível nacional em relação aos atletas que competiam em nível regional.

Constatou-se que atletas de modalidades individuais são mais motivados intrinsecamente e extrinsecamente do que atletas de modalidades coletivas. Porém, em relação à amotivação, não houve diferença entre os grupos. Justificamos este resultado pelo fato de que no Brasil, atletas de modalidades individuais, como natação e judo possuem maior número de praticantes e mais possibilidades de melhora de rendimento individual no seu desporto, contribuindo para aumentar a MI e a ME. Já atletas de modalidades coletivas (excluindo o futebol), tal como andebol e o basquetebol possuem pouco incentivos, além dos poucos clubes que oferecem estrutura para tal prática, diminuindo assim a possibilidade de uma maior motivação, seja intrínseca ou extrínseca.

No estudo de Gillet (2008), a amostra foi classificada da seguinte maneira: atletas de nível distrital, regional e nacional. A MI foi maior no grupo de nível nacional, bem como a ME injetada e regulação externa. Gillet (2008) também classificou a amostra por modalidade (coletivas e individuais), porém não observou nenhuma diferença estatisticamente significativa para as dimensões da motivação.

Murcia, Blanco, et al. (2007) compararam atletas jovens de modalidades coletivas e individuais. Os de modalidade individual foram positivamente associados ao grupo de perfil motivacional autoderminado, ou seja, maior MI, enquanto os atletas de equipas coletivas foram positivamente associados ao perfil menos autodeterminado e não autodeterminado (maior amotivação).

Lemyre et al. (2007) analisaram se a motivação autodeterminada poderia predizer sintomas de overtraining e burnout em atletas de elite, comparando para o efeito atletas de elite nível júnior com atletas de elite nível Olímpico. Os resultados obtidos indicaram diferenças significativas para todas as dimensões da motivação extrínseca e amotivação, sendo estas maiores nos atletas de elite júnior.

Os profissionais que lidam com jovens atletas deveriam ficar atentos aos comportamentos motivacionais de seus atletas e incentivar a prática por motivos intrínsecos. De acordo com Cresswell e Eklund (2005) a MI está negativamente associada ao burnout e ao abandono precoce de jovens atletas, enquanto a ME está diretamente ligada a esses fenómenos.

Na presente investigação, o SMS foi o único questionário aplicado nos atletas, enquanto na maioria das pesquisas realizadas com o SMS houve um segundo questionário para analisar outras comparações e correlações. Porém, justificamos por acreditar que o SMS já é por si só um questionário que analisa a motivação de 
uma forma ampla respondendo aos objetivos do presente estudo.

Diversos fatores podem ter afetado diretamente a motivação, gerando certa diferença em relação as outras pesquisas, tais como: diferenças socioeconómicas, culturais, tempo de prática no desporto e a idade. Além do mais podemos considerar como limitações da presente pesquisa o fato de não termos controlados as respostas "socialmente desejáveis", e o fato de termos colhidos os questionários em momentos diferentes (competições e treinamentos). Outro fator importante e que também não controlamos foi a influência do treinador na motivação dos atletas.

\section{CONCLUSÕES}

O atleta brasileiro possui características motivacionais únicas quando comparados com atletas de diferentes países. Neste sentido, índices de motivação autodeterminada elevados são encontrados em atletas brasileiros de modalidades individuais, homens, nível nacional e acima dos 25 anos. Enquanto mulheres, de equipas coletivas, nível regional e abaixo dos 25 anos obtiveram maiores índices de motivação pouco autoderminada. Estes dados podem servir de suporte para que os profissionais do desporto realizem intervenções em um dos mais importantes fatores psicológicos do rendimento desportivo: a motivação.

A partir do presente estudo, sugerimos futuras pesquisas com atletas brasileiros analisando uma maior amostra de atletas competindo em nível internacional, podendo ser realizado também trabalhos comparando com atletas de outros países. Além do mais, seria interessante realizar correlações entre as dimensões da motivação com outras variáveis psicológicas, como vimos na maioria das pesquisas, tais como o flow feeling, estratégias de coping ou para predizer sintomas de abandono e overtraining em atletas brasileiros.

Agradecimentos:

Nada a declarar.
Conflito de Interesses:

Nada a declarar.

Financiamento:

Nada a declarar.

\section{REFERÊNCIAS}

Alexandris, K., Tsorbatzoudis, C., \& Grouios, G. (2002). Perceived constraints on recreational sport participation: Investigating their relationship with intrinsic motivation, extrinsic motivation and amotivation. Journal of Leisure Research, 34, 233-252.

Amorose, A. J., \& Anderson-Butcher, D. (2007). Autonomy-supportive coaching and self-determined motivation in high school and college athletes: A test of self-determination theory. Psychology of Sport and Exercise, 8(1), 654-670. doi: 10.1016/j.psychsport.2006.11.003

Amorose, A. J., \& Horn, T. S. (2000). Intrinsic motivation: Relationship with collegiate athletes' gender, scholarship status, and perceptions of their coaches' behavior. Journal of Sport $\mathcal{E}$ Exercise Psychology, 22 (1), 63-84.

Bara Filho, M. G., Andrade, D., Miranda, R., Nuñez, J. L., Martin-Albo, J., \& Ribas, P. R. (2011). Preliminary validation of a Brazilian version of the sport motivation scale. Universitas Psicologica, 10(2), 363-372.

Chantal, Y., Guay, F., Dobreva-Martinova, T., \& Vallerand, R. J. (1996). Motivation and elite performance: An exploratory investigation with Bulgarian athletes. International Journal of Sport Psychology, 27(1), 173-182.

Coimbra, D., Gomes, S., Carvalho, F., Ferreira, R., Guillen, F., \& Miranda, R. (2008). O papel da psicologia do esporte para atletas e treinadores. Revista Brasileira de Ciência e Movimento, 16, 1-21.

Cresswell, S., \& Eklund, R. (2005). Motivation and burnout in professional rugby players. Research Quarterly for Exercise and Sport, 76(1), 370-376.

Deci, E., \& Ryan, R. (1985). Intrinsic motivation and self-determination in human behavior. New York: Plenum.

Fernandes, H. M., \& Vasconcelos-Raposo, J. (2005). Continuum de auto-determinação: Validade para a sua aplicação no contexto desportivo. Estudos de Psicologia, 10(1), 385-395. doi: 10.1590/S1413-294X2005000300007 
Fortier, M., Vallerand, R., Brière, N., \& Provencher, P. (1995). Competitive and recreational sport structures and gender: A test of their relationships with sport motivation. International Journal of Sport Psychology, 26(1), 24-39.

Gillet, N. (2008). Basic need satisfaction and motivation in sport. Athletic Insight, 11.

Gomes, S., Coimbra, D., Garcia, F., Miranda, R., Bara Filho, M. (2007). Análise da produção Científica em Psicologia do Esporte no Brasil e no Exterior. Revista Iberoamericana de Psicología Del Ejercicio y El Deporte, 2(1), 25-40.

Halbrook, M., Blom, L., Hurley, K., Bell, R., \& Holden, J. (2012). Relationships among motivation, gender, and, cohesion in a sample of collegiate athletes. Journal of Sport Behavior, 35, 61-77.

Kingston, K. M., Horrocks, C. S., \& Hanton, S. (2006). Do multidimensional intrinsic and extrinsic motivation profiles discriminate between athlete scholarship status and gender? European Journal of Sport Science, 6(1), 53-63.

Krinanthi, G., Konstantinos, M., \& Andreas, G. (2010). Self-determination and sport commitment: An evaluation by university intramural participants. International Journal of Fitness, 6(1), 41-52.

Lemyre, P., Roberts, G. C., \& Stray-Gunderrsen, J. (2007). Motivation, overtraining, and burnout in elite athletes. European Journal of Sport Science, $7(1), 115-126$.

Lujan, J. F. G., \& Deval, V. C. (2006). Psychological mediators and sport motivation in Spanish judokas. International Journal of Sport Science, 2(5), 1-11. doi: 10.5232/ricyde2006.00501

Martín-Balbo, J., Nuñes, J., Navarro, J., Leite, M., Almirón, M., \& Glavinich, N. (2007). Propriedades psicométricas de la version española de la escala de motivación deportiva en Paraguay. Revista Mexicana de Psicologia, 24(1), 43-52.

Miranda, R., \& Bara Filho, M. G. (2008). Construindo um atleta vencedor: Uma abordagem psicofísica do esporte. Porto Alegre: Artmed.

Murcia, J. A., \& Coll, D. G. (2006). A permanência de praticantes em programas aquáticos baseada na Teoria da Autodeterminação. Fitness $\mathcal{E}$ Performance Journal, 5(1), 5-9.

Murcia, J. A., Blanco, M. L., Galindo, C. M., Villodre, N. A., \& Coll, D. G. (2007). Efeitos do gênero, idade, e a frequência de pratica na motivação e o desfrute do exercício físico. Fitness $\mathcal{E}$ Performance Journal, 6(3), 140-146.

Murcia, J. A., Gimeno, E., \& Coll, D. (2007). Analizando la motivacion en el deporte: Un estúdio a través de la teoria de la autodeterminación. Apuntes de Psicología, 25(1), 35-51.

Nuñes, J., Martín-Balbo, J., Navarro, J., \& González, V. (2006). Preliminary validation of a Spanish Version of the Sport Motivation Scale. Perceptual and Motor Skills, 102 (1), 919-930.

Pelletier L., Tuson, K. M., Fortier, M., Vallerand, R. J., Brière, N., \& Blais, M. R. (1995) Toward a New Measure of Intrinsic Motivation, Extrinsic Motivation, and Amotivation in Sports: The Sport Motivation Scale (SMS) Journal of Sport $e$ Exercise Psychology, 17(1), 35-53.

Pero, R., Amici, S., Benvenuti, C., Mingati, C., Capranica, L., \& Pesce, C. (2009) Motivation for sport participation in older Italian athletes: The role of age, gender and competition level. Sport Science Health, 5(1), 61-69.

Perreault, S., \& Vallerand, R. (2007) A test of selfdetermination theory with wheelchair basketball players with and without disability. Adapted Physical Activity Quarterly, 24, 305-316.

Ryan, R., \& Deci, E. (2000). Self-determination Theory and the facilitation of intrinsic motivation, social development, and well-being. American Psychologist, 55(1), 68-78. doi: 10. 103 7/110003-066X.55.1.68

Sarrazin, P., Vallerand, R. J., Guillet, E., Pelletier, L. G., \& Cury, F. (2002). Motivation and dropout in female handballers: A 21-month prospective study. European Journal of Social Psychology, 32(1), 395-418. doi: 10.1002/ejsp.98

Sena Junior, A. W. (2012). Motivação e Flow-Feeling na Corrida de Rua. Dissertação de Mestrado não publicada, Universidade Federal de Juiz de Fora, Minas Gerais, Brasil.

Silva, R., Matias, T., Viana, M., \& Andrade, A. (2012). Relação da prática de exercícios físicos e fatores associados às regulações motivacionais de adolescentes brasileiros. Motricidade, 8(2), 8-21. doi: 106063/motricidade.8(2).708

Vallerand, R., Pelletier, L., Blais, M., Brière, N., Senécal, C., \& Vallières, E. (1992). The academic motivation scale: A measure of intrinsic, extrinsic, and amotivation in education. Educational and Psychological Measurement, 52, 1003-1019.

(c) EY-No Todo o conteúdo da revista Motricidade está licenciado sob a Creative Commons, exceto quando especificado em contrário e nos conteúdos retirados de outras fontes bibliográficas. 